\title{
Deformable Registration of Tumor-Diseased Brain Images
}

\author{
Tianming Liu, Dinggang Shen, and Christos Davatzikos \\ Section of Biomedical Image Analysis, Department of Radiology, \\ University of Pennsylvania, Philadelphia, PA 19104 \\ \{tliu, dgshen, christos\} @rad.upenn.edu
}

\begin{abstract}
This paper presents an approach for deformable registration of a normal brain atlas to visible anatomic structures in a tumor-diseased brain image. We restrict our attention to cortical surfaces. First, a model surface in the atlas is warped to the tumor-diseased brain image via a HAMMER-based volumetric registration algorithm. However, the volumetric warping is generally inaccurate around the tumor region, due to the lack of reliable features to which the atlas can be matched. Therefore, the model structures for which no reliable matches are found are labeled by a Markov Random Field-Maximum A Posteriori approach. A statistically-based interpolation method is then used to correct/refine the volumetric warping for those structures. Finally, with the good initialization obtained by the above steps and the identification of the part of the model anatomy that can be recognized in the patient's image, the model surface is adaptively warped to its counterpart that is visible in the tumordiseased brain image through a surface registration procedure. Preliminary results show good performance on both simulated and real tumor-diseased brain images.
\end{abstract}

\section{Introduction}

In neurosurgical planning, it is of interest to register a normal brain atlas to a patient's tumor-diseased brain image, in order to transfer the information available in the atlas to the patient's space for surgical guidance. In this paper, we propose an approach for deformable registration of a normal brain atlas to visible anatomic structures in a patient's brain image in the presence of tumor. We assume that only part of the patient's anatomy is visible, since some tissue might be severely distorted by the tumor and/or obscured by edema, and some tissue might have died during the tumor growth. Our long-term goal is to use the registered visible anatomy to estimate 1) the tumor location in a standardized atlas for the purpose of atlas construction from such patients and 2) the location of deformed and invisible structures for the purpose of neurosurgical planning. As an example, Figure 1 shows a normal atlas and a tumordiseased brain image to be registered.

In general, the proposed approach extracts and exploits reliable information to drive the registration. There are four steps in our approach, as summarized in Figure 2. Firstly, a complete model surface (i.e., see Figure 1) in the atlas is warped to the space of a patient's brain by a HAMMER-based volumetric registration algorithm [6]. Secondly, a confidence level for the accuracy of volumetric warping for each model 
surface vertex is determined by computing the possibility of this vertex has found its counterpart in the tumor-diseased brain. The degree of confidence will be low around the region where image features are missing due to the presence of tumor and/or edema. Based on the confidence map, we employ a Markov Random Field (MRF)Maximum A Posteriori (MAP) approach to classify the model surface vertices into two groups, one roughly corresponding to the missing/invisible anatomy in the patient's brain, and the other roughly corresponding to the visible anatomy. Thirdly, a statistically-based interpolation method is used to correct/refine the deformation fields in the low confidence regions by those in the relatively high confidence regions. Finally, with the good initialization obtained by the first three steps and the identification of the part of the model anatomy that can be recognized in the patient's image, the model surface is adaptively warped to its counterpart visible in the patient's brain image by a surface registration procedure.

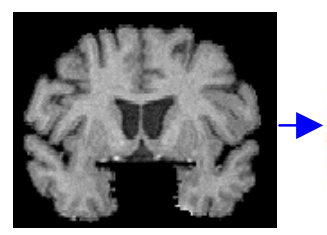

(a)

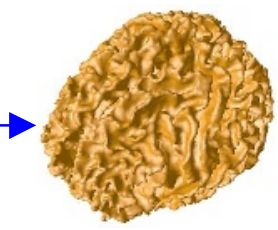

(b)

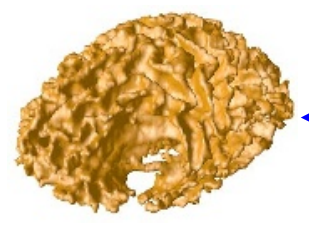

(c)

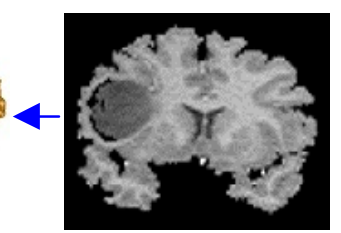

(d)

Fig. 1. A normal atlas and a tumor-diseased brain image, to be registered. (a) A slice of the normal atlas. (b) The WM/GM surface reconstructed from the atlas. (c) The WM/GM surface reconstructed from a tumor-diseased brain image. (d) A typical slice of the tumor-diseased brain image (low grade glioma).

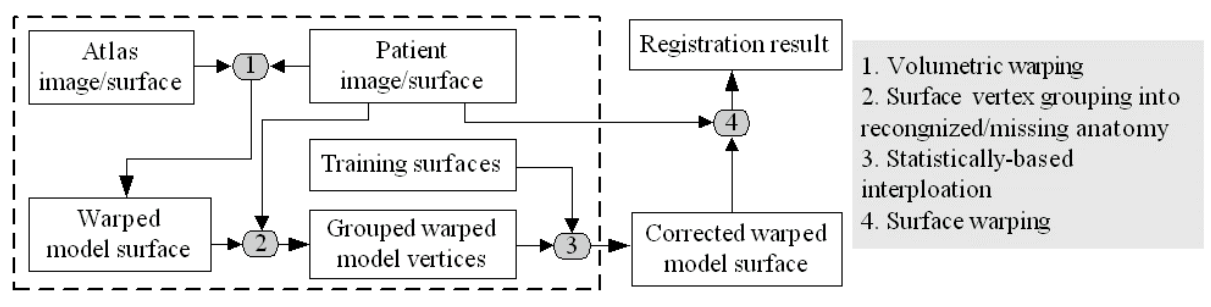

Fig. 2. The summary of our registration approach. The steps in the dashed block provide the initialization for the model surface in the atlas, to be adaptively deformed in step 4.

\section{Method}

\subsection{Volumetric Warping}

The HAMMER-based volumetric registration algorithm [6] is used to bring the model surface in the atlas close to its counterpart visible in the tumor-diseased brain image, in order to facilitate the subsequent steps. The registration accuracy is expected to be relatively high in normal brain regions [6]. However, the volumetric warping is expected to be less accurate around the tumor region, due to the lack of reliable features to which the atlas can be matched. To address this problem, a statistically- 
based interpolation method is used in Section 2.3 to correct/refine the volumetric warping around the tumor region, in order to provide better initialization for the model surface so that it can be registered with visible structures in the tumor-diseased brain through a surface registration described in Section 2.4.

\subsection{Surface Vertex Grouping}

To facilitate the statistically-based interpolation in Section 2.3, which requires hard grouping of the model surface vertices, the warped model vertices are classified into two groups. One group includes the vertices for which a good match can be found in the patient's brain after volumetric warping, and the opposite for the second group.

Firstly, we compute the confidence level of volumetric warping for each model surface vertex, in order to roughly measure the warping accuracy. The confidence level is defined as the similarity between this model surface vertex and its most likely counterpart in the surface of the tumor-diseased brain. Intuitively, if the similarity is higher for a model vertex, we can have more chance of detecting its correspondence in the registration procedure and thus have more confidence in the accuracy of the volumetric warping for this model vertex. The similarity between a model surface vertex $s_{i}^{\text {Mdl }}$ and a surface vertex in the patient's brain $s_{j}^{\text {Ptn }}$ is defined as:

$$
f\left(s_{i}^{\mathrm{Mdl}}, s_{j}^{\mathrm{Ptn}}\right)=\left(n\left(s_{i}^{\mathrm{Mdl}}\right) \bullet n\left(s_{j}^{\mathrm{Ptn}}\right)\right) \cdot C\left(s_{i}^{\mathrm{Mdl}}, s_{j}^{\mathrm{Ptn}}\right)
$$

where $n\left(s_{i}^{\mathrm{Mdl}}\right)$ is a normal vector of the vertex $s_{i}^{\mathrm{Mdl}}$, ' $\bullet$ ' denotes the dot product of two vectors, and $C\left(s_{i}^{\mathrm{Mdl}}, s_{j}^{\mathrm{Ptn}}\right)$ is the similarity of the surface curvatures of vertex $s_{i}^{\mathrm{Mdl}}$ and $s_{j}^{\mathrm{Ptn}}$, which is defined next:

$$
C\left(s_{i}^{\mathrm{Mdl}}, s_{j}^{\mathrm{Ptn}}\right)=1-\left\|c\left(s_{i}^{\mathrm{Mdl}}\right)-c\left(s_{j}^{\mathrm{Ptn}}\right)\right\| / /\left(c\left(s_{i}^{\mathrm{Mdl}}\right)+c\left(s_{j}^{\mathrm{Ptn}}\right)\right)
$$

where $c(\bullet)$ is calculated as $\sqrt{\left(k_{1}^{2}+k_{2}^{2}\right) / 2}$, with $k_{1}$ and $k_{2}$ as two principle curvatures. For each model surface vertex $s_{i}^{\mathrm{Mdl}}$, we look for a surface vertex in the patient's brain image with the largest degree of similarity. Here, $y_{s_{i}^{\text {Mu }}}$ is used to denote the largest degree of similarity found for the model surface vertex $s_{i}^{\text {Mdl }}$. These computations create the confidence map, denoted by $Y=\left\{y_{s_{i}^{\text {Mal }}}\right\}$.

To classify model surface vertices into two groups based on above confidence map, we employ a MRF-MAP method, because MRF theory provides an efficient and powerful framework for modeling spatial dependence and MAP estimation offers a mechanism for finding a desirable solution. Let $S^{\mathrm{Mdl}}=\left\{s_{i}^{\mathrm{Mdl}}\right\}$ denote the set of model vertices. For each vertex, a first-order neighborhood system, consisting of its immediate neighbors, is defined for the MRF. Let $\Lambda=\{0,1\}$ be the label set, where 0 means recognized/matched vertex and 1 means unrecognized/missing vertex. Let $\omega=\left\{\omega_{s_{i}^{\text {Mal }}}\right\}$ be the vertex grouping result, where $\omega_{s_{i}^{\text {Mal }}}$ takes a value from $\Lambda$. Now, the objective of the grouping is to maximize the posteriori probability of $P(\omega \mid Y)$. According to the Bayes theory, the MAP-based vertex grouping is obtained by: 


$$
\omega^{*}=\underset{\omega \in \Omega}{\arg \max }(P(Y \mid \omega) P(\omega))
$$

where $\Omega$ is the set of all possible grouping results. The term $P(Y \mid \omega)$ is modeled as an independent multivariate Gaussian distribution. According to the HammersleyClifford theorem [1], $P(\omega)$ has the Gibbs distribution, and its energy term is the sum of clique potentials over all possible cliques. Here, only cliques of a size up to two are considered, and the homogeneous multi-level logistic (MLL) distribution [1] is used to define the potential for two adjacent vertices. To solve Eq. (3), we use the local optimization method of Iterated Conditional Modes (ICM) [2].

Notably, we reconstruct anatomic surface in the patient's brain directly from the WM/GM volume in the tissue-classified image [4], without pre-segmentation of the tumor. This may introduce false WM/GM structures when performing the marching cubes algorithm to the possible extra WM/GM boundaries, produced during the tissue classification of the tumor-diseased brain image. However, those false structures have little effect on the surface vertex grouping and the following step of surface registration, since no structures of the model would match them.

\subsection{Statistically-Based Interpolation}

The general idea of the statistically-based interpolation is that we determine the deformation vectors of part of model surface by those of the remainder of the model surface using a statistical estimation method. In this paper, we use the statisticallybased interpolation to correct/refine the volumetric warping in the low confidence regions.

More specifically, we discard the volumetric warping in the low confidence regions $\left\{s_{i}^{\mathrm{Mdl}}, \omega_{s_{i}^{\text {Mdl }}}^{*}=1\right\}$, and estimate it by that in the high confidence regions $\left\{s_{i}^{\mathrm{Mdl}}, \omega_{s_{i}^{\text {Mdl }}}^{*}=0\right\}$, using a statistical estimation method based on canonical correlation analysis in [5]. The statistical estimation is achieved by learning from a training set of deformation fields, which is obtained by warping a model surface in the atlas into individual normal brain images via the hybrid registration method in $[3,4]$.

It is worth noting that the statistically-based interpolation places the model structures much closer to their counterparts visible around the tumor region than the volumetric warping in Section 2.1, thus providing a good start for the next step of surface registration. In the future, we intend to take into account the statistics of the tumor mass effect, which can be obtained via a large number of tumor growth simulations at different brain locations $[8,9]$, in the statistically-based interpolation, thus leading to better initialization for the following step of surface warping.

\subsection{Surface Warping}

Based on the initialization obtained by the first three steps, the surface warping step is designed to adaptively deform the model surface $S^{\mathrm{Mdl}}=\left\{s_{i}^{\mathrm{Mdl}}\right\}$ to the surface in the patient's tumor-diseased brain image $S^{\mathrm{Ptn}}=\left\{s_{i}^{\mathrm{Ptn}}\right\}$, by using the above-collected confidence map and vertex grouping result as deformation adaptation knowledge to 
guide the surface registration. Notably, we allow only the model vertices with high confidence levels to search for their correspondences in the surface of the tumordiseased brain image. For any model vertex $s_{i}^{\text {Mdl }}$, if that vertex finds its correspondence, then its surface patch $P\left(s_{i}^{\mathrm{Mdl}}, r\right)$ within a geodesic distance $r$ will be deformed to the patient's brain surface by a local transformation $T_{s_{i}^{\text {Mul }}}$. Therefore, the whole transformation $h$ for the warping of the model surface is decomposed into a number of local transformations $\left\{T_{s_{i}^{\text {Mal }}}\right\}$, i.e., $h=\left\{T_{s_{i}^{\text {Mal }}}\right\}$.

Mathematically, the surface warping can be formulated as a procedure of minimizing the energy function as defined next:

$$
E\left(h=\left\{T_{s_{i}^{\text {Mal }}}\right\}\right)=\sum_{s_{i}^{\text {Mal }} \in S^{\text {Matl }}, \omega_{s_{i}^{\text {Mal }}}=0} y_{s_{i}^{\text {Mal }}}\left(w_{\text {Ext }} E_{s_{i}^{\text {Mal }}}^{\text {Ext }}\left(T_{s_{i}^{\text {Mal }}}\right)+w_{\text {Int }} E_{s_{i}^{\text {Mul }}}^{\text {Int }}\left(T_{s_{i}^{\text {Mal }}}\right)\right)
$$

where $y_{s_{i} \text { ad }}$ is the confidence level of $s_{i}^{\text {Mdl }}$ and used to weight different model vertices differently. Only model vertices with high confidence levels, namely having $\omega_{s_{i}^{\text {Mul }}}=0$, are considered as viable to deform the model surface. $w_{\text {Ext }}$ and $w_{\text {Int }}$ are the weights for external and internal energies, respectively.

The external energy $E_{s_{i}^{\text {Mal }}}^{\mathrm{Ext}}\left(T_{s_{i}^{\text {Mall }}}\right)$ measures the similarity of the surface patches in the atlas and those in the patient, respectively. It requires that the curvature of the neighboring vertex $s_{j}^{\mathrm{Mdl}}$ in the model surface patch $P\left(s_{i}^{\mathrm{Mdl}}, r\right)$ be similar to that of its counterpart in the patient, and also that the normal direction of vertex $s_{j}^{\text {Mdl }}$ be close to that of its counterpart. The mathematical definition is given as:

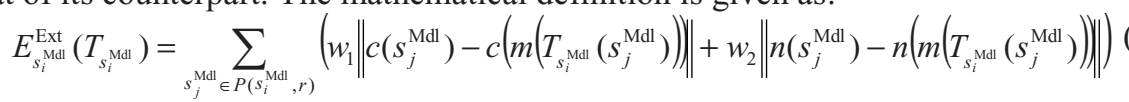

where $c(\cdot)$ and $n(\cdot)$ denote the surface curvedness and the normal vector, respectively. $m($.$) denotes a mapping of a vertex to the closest vertex in the patient's$ surface, since the transformed model vertex $T_{s_{i}^{\text {Mal }}}\left(s_{j}^{\mathrm{Mdl}}\right)$ is not necessary on the patient's surface vertex. $w_{1}$ and $w_{2}$ are weighting parameters.

The internal energy term $E_{s_{i}^{\text {all }}}^{\mathrm{Int}}\left(T_{s_{i}^{\text {atl }}}\right)$ is defined as the same as that in [3, 4], in order to preserve the shape of the model surface during deformation. To minimize the energy function, we use a greedy deformation algorithm. Similarly, we use the scheme in [4] to prevent self-intersection during deformation, and use the adaptive deformation strategy $[4,7]$ based on the confidence map to reduce the chances of being trapped of local minima. Notably, the confidence map and vertex grouping result are updated using the method in Section 2.2 after each iteration of the energy minimization process, since during the surface deformation procedure, some model structures might move outside the tumor, while other structures might move inside it.

So far, we have not used any tumor information in the registration. However, integrating tumor information, i.e., the location of tumor, into the registration procedure can potentially improve the registration accuracy. 


\section{Results}

The performance of the proposed approach is tested by both simulated and real tumordiseased brains. In these experiments, we register only WM/GM surfaces.

In this experiment using simulated tumor-diseased image, we compare the registration result obtained by our proposed approach with the ground-truth that is created as follows. We employ the method in [9] to grow a tumor in a normal brain image, and produce a tumor-induced deformation field. Then, the model WM/GM surface in the atlas is registered with its counterpart in the normal brain image [3, 4], and further deformed by the tumor-induced deformation field generated in the tumor simulation. The finally deformed model WM/GM surface is regarded as the ground-truth. Independently, we use the proposed approach to register the WM/GM surface reconstructed from the simulated tumor-diseased brain image. Figure 3 shows the histogram of registration errors of the proposed approach, compared to the groundtruth, after initialization and after final surface warping. The average registration error in the visible structures of the tumor-diseased brain drops from $1.4 \mathrm{~mm}$ to $1.1 \mathrm{~mm}$, from initialization to final surface warping. As for the WM/GM surface around the tumor region, the average registration error drops from $2.5 \mathrm{~mm}$ to $1.8 \mathrm{~mm}$. Figure 4 shows the overlay of a typical slice with the patient's WM/GM surface, the initialized model surface and the finally warped model surface. The registration result seems visually reasonable.

The performance of the proposed approach is also tested using real tumor-diseased brains obtained from the SPL brain tumor database [10]. Figure 5 shows the overlay of a typical slice with three surfaces, a patient's WM/GM surface, the initialized model surface and the finally warped model surface. It can be seen that the registration result is visually reasonable. For reference, the patient's WM/GM surface can be found in Figure 1. Since we do not know the true correspondences among the $\mathrm{WM} / \mathrm{GM}$ surfaces in this experiment, we used the surface distance to measure the registration accuracy. By this measurement, the average distance is $0.3 \mathrm{~mm}$ from the finally warped model surface to the patient's surface, and is $0.46 \mathrm{~mm}$ from the patient's surface to the finally warped model surface. Here, only regions labeled high confidence are counted in the surface distance calculation. Notably, the average

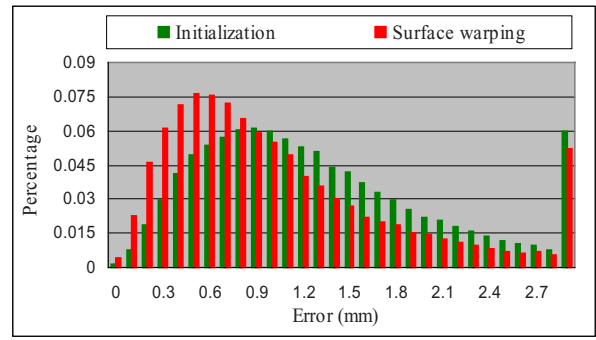

(a)

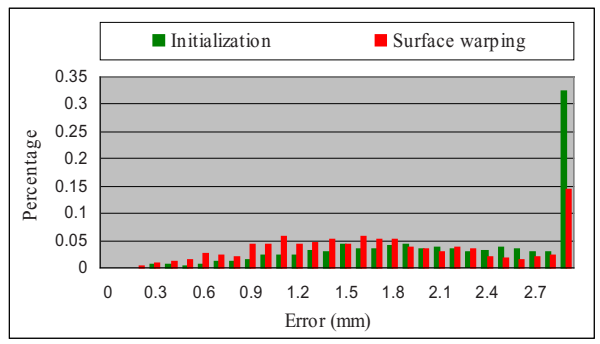

(b)

Fig. 3. Histogram of registration errors, after initialization and after final surface warping. The horizontal axis is the registration error, and the vertical axis is the percentage of the vertices on the model surface. (a) For all visible WM/GM structures. (b) Only for the visible WM/GM structures around the tumor. 


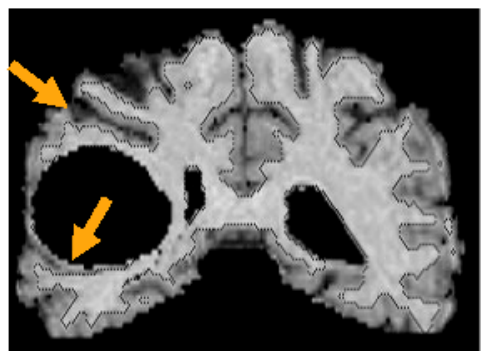

(a)

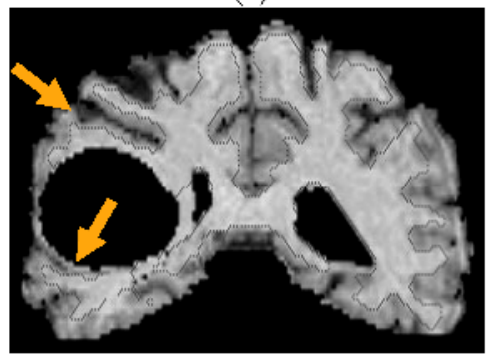

(c)

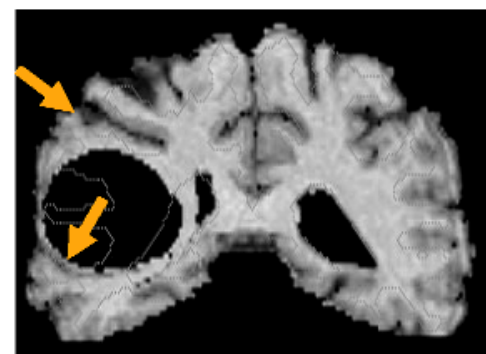

(b)

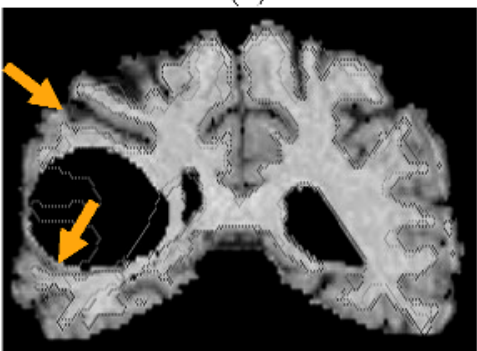

(d)

Fig. 4. Registration result for a simulated tumor-diseased brain. (a) Overlay of a typical slice and its own WM/GM surface. (b) The initialized model WM/GM surface. (c) The finally warped model WM/GM surface. (d) Overlay of all three surfaces. The arrows indicate the positions where the registration results were significantly improved by the surface warping.

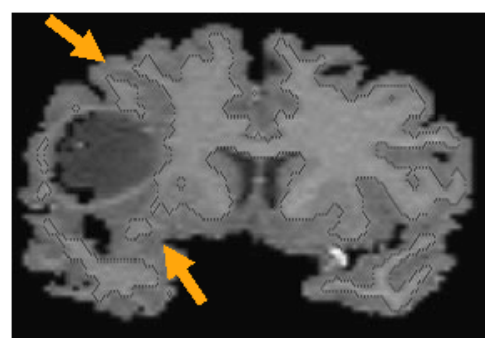

(a)

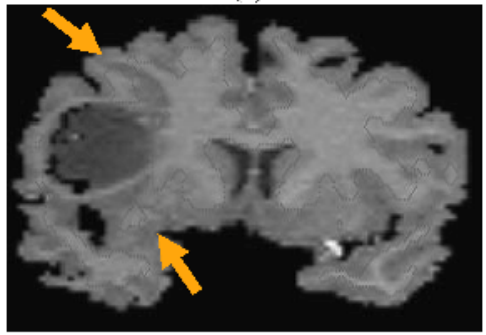

(c)

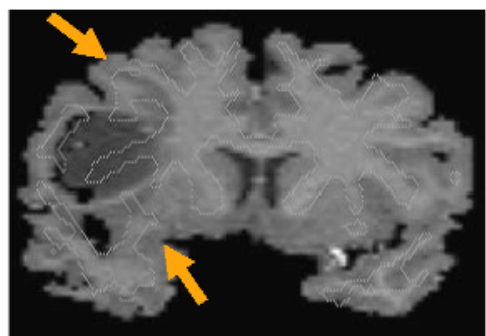

(b)

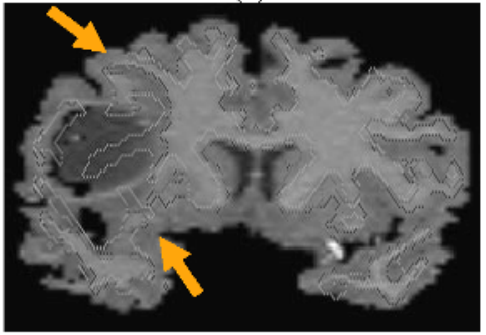

(d)

Fig. 5. Registration result for a real tumor-diseased brain. (a) Overlay of a typical slice and its own WM/GM surface. (b) The initialized model WM/GM surface. (c) The finally warped model WM/GM surface, shown only on visible WM/GM structures of tumor brain. (d) Overlay of all three surfaces. 


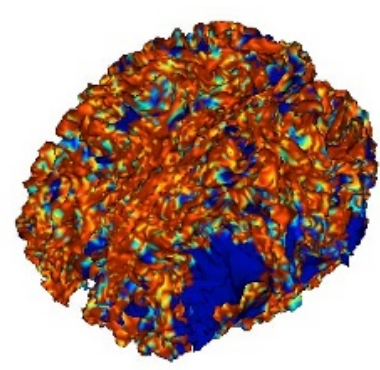

(a)

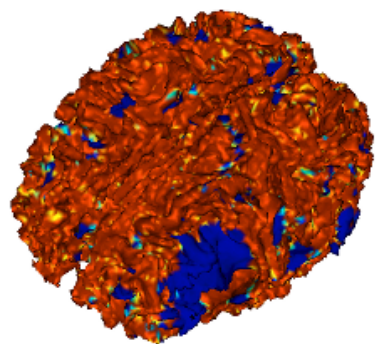

(b)

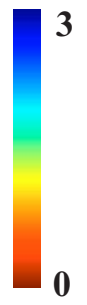

Fig. 6. Color-coded map of surface distance $(\mathrm{mm})$, according to the color-bar at the right. (a) After initialization. (b) After final surface warping.

model-to-patient surface distance drops 57 percent after the surface warping. This can be confirmed by a color-coded map of distances of the warped model surface to the patient's surface in Figure 6.

\section{Conclusion}

We proposed an approach for deformable registration of a normal atlas to the visible anatomic structures in a tumor-diseased brain. Although we registered only WM/GM surface in this paper, our approach can be applied to register other surfaces in a tumor-diseased brain, including the GM/CSF surface. In the future, we intend to integrate into the registration approach the statistics of the mass effect of a tumor, which are determined via a large number of tumor growth simulations at different brain locations, and validate the approach using a larger number of patient brains.

Acknowledgement. The authors would like to thank Ashraf Mohamed for providing the simulated tumor-diseased brain and the SPL group of the Brigham and Women's Hospital for sharing the brain tumor database.

\section{References}

1. Stan Z. Li, Markov random field modeling in image analysis, Springer-Verlag New York, Inc., Secaucus, NJ, 2001.

2. J. Besag, "On the statistical analysis of dirty pictures," J. Roy. Statist. Soc. B 48(3), pp. 259-302, 1986.

3. T. Liu, D. Shen, C. Davatzikos, "Deformable registration of cortical structures via hybrid volumetric and surface warping," MICCAI 2003, Canada, 2003.

4. T. Liu, D. Shen, C. Davatzikos, "Deformable registration of cortical structures via hybrid volumetric and surface warping," submitted to NeuroImage.

5. T. Liu, D. Shen, C. Davatzikos, "Predictive modeling of anatomic structures using canonical correlation analysis," To appear, ISBI 2004, April 15-18, Arlington, VA.

6. D. Shen and C. Davatzikos, "HAMMER: hierarchical attribute matching mechanism for elastic registration,” IEEE Transactions on Medical Imaging, 21(11): 1421-1439, 2002. 
7. D. Shen, E. H. Herskovits, and C. Davatzikos, "An adaptive-focus statistical shape model for segmentation and shape modeling of 3D brain structures," IEEE Transactions on Medical Imaging, 20(4): 257-270, April 2001.

8. C. Davatzikos, D. Shen, A. Mohamed, and S. Kyriacou, "A framework for predictive modeling of anatomical deformations," IEEE TMI, 20 (8):836-843, 2001.

9. A. Mohamed and C. Davatzikos, "Finite element mesh generation and remeshing from segmented medical images," To appear, ISBI 2004, Arlington, VA, 2004.

10. M. Kaus, S. K. Warfield, A. Nabavi, P. M. Black, F. A. Jolesz, and R. Kikinis. "Automated segmentation of MRI of brain tumors," Radiology, 218:586-591, 2001. 